\title{
Profound exercise-induced ST segment depression in a patient with Wolff-Parkinson-White syndrome and normal coronary arteriograms
}

\author{
PHILIP GREENLAND, ROBERT KAUFFMAN, AND E KENNETH WEIR \\ From the Division of Cardiology, Veterans Administration Medical Center and the University of Minnesota, \\ Minneapolis, Minnesota
}

The electrocardiogram is notoriously unreliable for the diagnosis of ischaemic heart disease in patients with Wolff-Parkinson-White syndrome. Abnormalities in the resting tracing may lead to false positive diagnoses of inferior or anterior myocardial infarction. Although ST segment depression with exercise has been reported in patients with Wolff-Parkinson-White syndrome, the depth of depression has been modest ( $2 \mathrm{~mm}$ or less). 1

In the present report we describe marked ST segment depression $(>4 \mathrm{~mm})$ during exercise in a patient with Wolff-Parkinson-White syndrome who was shown subsequently to have normal coronary anatomy by arteriography and normal myocardial function by resting and exercise radionuclide angiography.

\section{Case report}

A 53-year-old white fireman was referred for evaluation of exercise-induced chest pain. He had been in good health until two weeks before admission when he first experienced an episode of substernal chest discomfort while operating heavy equipment. The pain lasted five minutes, did not radiate, had no associated symptoms, and was relieved by rest. A similar episode occurred the next day, and he went to his family physician.

Coronary risk factors included a strong family history, several uncles and a brother having died before the age of 50 years of myocardial infarction or unexplained sudden death, and his father having died of myocardial infarction aged 66 years. The patient was moderately obese. Hyperglycaemia was noted on routine screening six months before this illness. He was a non-smoker, without hypertension or hyperlipidemia.

Type A Wolff-Parkinson-White syndrome had previously been detected by routine electrocardiography. He denied palpitations, tachycardia or faints and had never required any drugs. Physical examination demonstrated only moderate obesity (weight $90 \mathrm{~kg}$ ). Cardiovascular examination was entirely normal.

Address for reprint requests: Dr E Kenneth Weir, Veterans Administration Medical Center, Cardiovascular Section, Minneapolis, Minnesota 55417, USA.
Treadmill exercise testing was performed using the Bruce protocol because of the suspicion of coronary artery disease. Exercise was terminated by the physician after four minutes and 10 seconds because of markedly abnormal ST segment depression (figure). The heart rate reached 146 and blood pressure peaked at 190/100. The patient denied chest discomfort during the test. ST-T wave changes did not resolve completely for 10 minutes.

Resting and exercise radionuclide angiograms were performed and revealed a resting ejection fraction of $48 \%$, increasing to $58 \%$ with exercise. Again, no chest pain occurred.

Coronary arteriography was performed by Judkin's technique. No atherosclerotic obstructions were detected at rest. The final diagnosis was chest pain of uncertain aetiology in association with normal coronary arteries.

\section{Discussion}

The exercise electrocardiogram has enjoyed a lengthy period of use in the detection and classification of coronary artery disease. However, its predictive value in diagnosing coronary artery disease is clearly affected by a number of factors, including the type of exercise-induced abnormality seen, ${ }^{2}$ the presence or absence of resting electrocardiographic abnormalities, $^{3}$ and the type of patient in whom the test is used. ${ }^{4}$ False positive tests also occur in patients on digitalis derivatives and in patients with left ventricular hypertrophy or left bundle branch block at rest. $^{3}$

A number of recent studies have emphasised measures capable of improving the predictive accuracy of exercise stress testing. In addition to exclusion of patients with pre-existent electrocardiographic abnormalities, other approaches have included the use of Bayesian analysis of coronary risk in conjunction with exercise electrocardiography in an effort to increase diagnostic accuracy. ${ }^{4}$ An additional factor considered useful is the degree of ST segment depression caused by exercise. Many now consider exercise-induced ST depression in excess of $3 \mathrm{~mm}$ to be a highly accurate predictor of severe, or critical, coronary artery disease. ${ }^{5}$ ST changes of this magnitude generally indicate extensive coronary disease or left main 

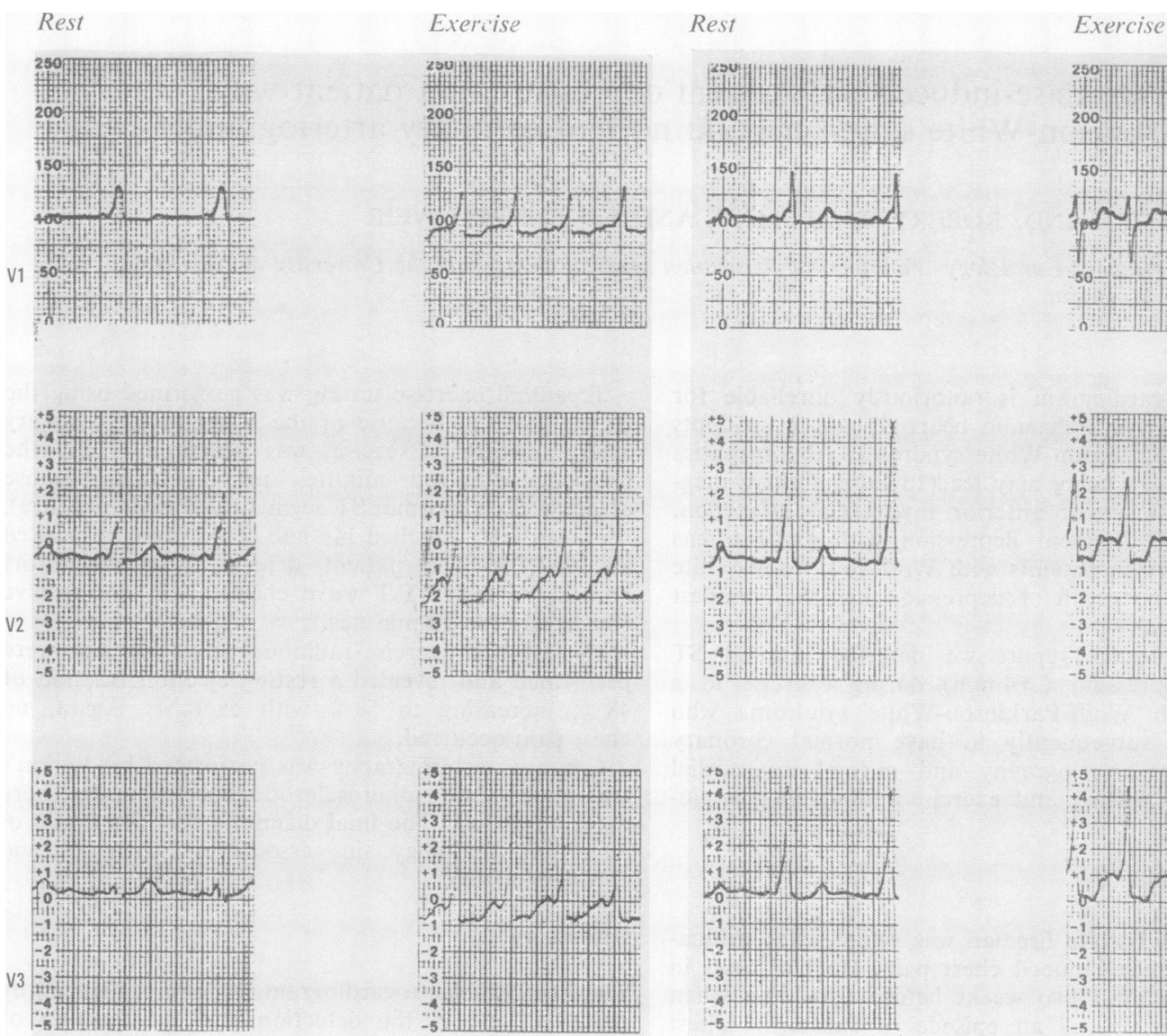

Figure Marked ST depression induced by four minutes of exercise.

coronary disease, and are also thought useful in excluding false positive diagnoses. However, the possibility that coincidental ischaemic heart disease might exacerbate the ST depression observed during exercise in patients with Wolff-Parkinson-White syndrome has not been discussed.

It is known that the exercise electrocardiogram may be falsely positive in patients with this syndrome. ${ }^{1}$ However, none of the patients in that report was noted to have severe ST depression and none had coronary arteriography to exclude definitively the presence of coronary artery disease. Therefore the patient presented here is unusual in both the magnitude of ST segment depression and the documentation of normal coronary arteriography. The normal radionuclide response to exercise in this patient suggests that this technique may provide an alternative to coronary arteriography in such patients. In addition the case demonstrates that even profound ST depression $(>3 \mathrm{~mm})$ cannot be used reliably to increase the predictive value of the exercise stress test in such patients.
It is a pleasure to acknowledge the co-operation $\oint_{0}$ Dr DL Hanson in the management of this patient.8

\section{References}

1 Gazes DC. False-positive exercise test in the presence of the Wolff-Parkinson-White syndrome. Am Heart J 1969; 78:13-5.

2 Ellestad MH, Wan MKC. Predictive implicatious of stress testing. Follow-up of 2700 subjects after maximal treadmill stress testing. Circulation 1975; 51:363-9.

3 Surawicz B, Saito S. Exercise testing for detection of myocardial ischemia in patients with abnormal electrocardiograms at rest. Am J Cardiol 1978; 41:943-51.

4 Diamond GA, Forrester JS. Analysis of probability as an aid in the clinical diagnosis of coronary artery disease. $N$ Engl J Med 1979; 300:1350-8.

5 Goldman S, Tselos S, Cohn K. Marked depth $\AA_{f} f$ ST segment depression during treadmill exercise testing. Indicator of severe coronary artezi disease. Chest 1976; 69:729-33. 